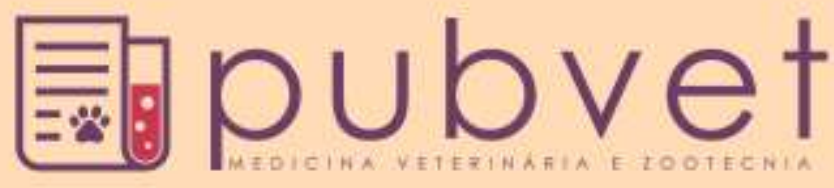

HTTP://DX.DOI.ORG/10.22256/PUBVET.VIIN3.243-255

\title{
Colagenases do pescado e suas aplicações industriais
}

\section{Vagne de Melo Oliveira ${ }^{1}$, Márcia Nieves Carneiro da Cunha ${ }^{1}$, Caio Rodrigo Dias Assis ${ }^{1}$, Thiago Pajeú Nascimento ${ }^{1}$, Polyanna Nunes Herculano ${ }^{1}$, Maria Taciana Holanda Cavalcanti $^{1}$, Ana Lúcia Figueiredo Porto ${ }^{1 *}$}

${ }^{1}$ Universidade Federal Rural de Pernambuco, Departamento de Morfologia e Fisiologia Animal, DMFA, Recife, PE, Brasil. E-mail: vagne_melo@hotmail.com;_marcianieves@yahoo.com.br; caiodias2@hotmail.com; thiago_pajeu@hotmail.com; polyanna.herculano@hotmail.com; mtcvsoares@yahoo.com.br;

*Autor para correspondência, E-mail: analuporto@yahoo.com.br.

\begin{abstract}
RESUMO. Colagenases são grupos de enzimas capazes de clivar a tripla-hélice do colágeno não tendo ação sobre outro tipo de proteína. São obtidas a partir de uma vasta gama de fontes, tais como tecido animal e vegetal. Uma fonte promissora na atualidade é a partir de resíduos negligenciados do processamento do pescado, tais como vísceras internas (intestino). Esta revisão objetivou realizar um levantamento quantitativo da produção cientifica relacionada à colagenase do pescado, bem como dissertar sobre as principais características bioquímicas e aplicações industriais dessa protease, com ênfase nas colagenases extraídas a partir de organismos aquáticos. Dessa forma, através de análise do Scopus, no período de 1963 a 2016, foram detectadas 274 citações ao termo "collagenase fish". A produção cientifica pertinente a colagenase do pescado está intrinsicamente relacionada a necessidade industrial de fontes alternativas dessas proteases colagenolíticas, devido sua extensa aplicação industrial, tais como nos segmentos alimentícios, biomédicos, terapêuticos e têxtil.
\end{abstract}

Palavras chave: Biotecnologia, enzimas, pescado, protease, resíduos

\section{Fish collagenases and its industrial applications}

\begin{abstract}
Collagenases are a group of enzymes capable of cleaving the triple-helix collagen, having no action on another type of protein. They are obtained from a wide range of sources, such as animal and plant tissue. A promising source at present is from neglected fish processing residues, such as internal viscera (intestine). This review aimed to quantify the scientific production related to fish collagenase, as well as to discuss the main biochemical characteristics and industrial applications of this protease, with emphasis on collagenases extracted from aquatic organisms. Thus, through analysis of Scopus, from 1963 to 2016, 274 citations were detected to the term "collagenase fish". The scientific production relevant to fish collagenase is intrinsically related to the industrial need for alternative sources of these collagenolytic proteases, due to their extensive industrial application, such as in the food, biomedical, therapeutic and textile segments.
\end{abstract}

Keywords: Biotechnology, enzymes, fish, protease, residues

\section{Colagenasas do pescado y sus aplicaciones industriales}

RESUMEN. Las colagenasas son grupos de enzimas capaces de escindir la triple hélice del colágeno sin acción sobre otro tipo de proteína. Se obtienen de una amplia variedad de fuentes, tales como tejidos de plantas y animales. Una fuente prometedora actualmente es a partir de residuos no utilizados del procesamiento de pescado, tales como las vísceras internas (intestino). Esta revisión tuvo como objetivo realizar un estudio cuantitativo de la 
producción científica relacionada con la colagenasa de pecescados, así como detalles sobre las principales características bioquímicas y las aplicaciones industriales de esa proteasa, con énfasis en la colagenasa extraída de los organismos acuáticos. De este modo, mediante el análisis de Scopus en 1963 a 2016, se detectaron 274 citas con el término "collagenase fish". La producción científica pertinente a colagenasa do pescado está intrínsecamente relacionada a la necesidad industrial de fuentes alternativas de estas proteasas colagenolíticas, debido a su amplia aplicación industrial, tales como en los segmentos de alimenticios, biomédicos, terapéutica y textiles.

Palabras clave: Biotecnología, enzimas, pescado, proteasa, residuos

\section{Introdução}

O mercado global de enzimas deve crescer em média 4,6\% até 2020, passando de US \$5,78 bilhões em 2015 para US \$ 7,2 bilhões em 2020. Este mercado inclui enzimas usadas em aplicações industriais (alimentos e bebidas, produtos de limpeza, a produção de biocombustíveis, ração animal e outros mercados) e aplicações especiais (pesquisa e biotecnologia, diagnósticos e biocatalisadores). Alimentos e bebidas continuarão a ser o maior mercado para as enzimas, com ganhos impulsionados pelo aumento do consumo de produtos que contêm enzimas em regiões em desenvolvimento (Bussiness Standard, 2016). O potencial brasileiro para uso de enzimas é bastante atraente para o setor agroindustrial, podendo gerar novos empregos e reduzir gastos com importação, uma vez que só em 2005 o país importou mais de $3.295 .845 \mathrm{~kg}$ de enzimas industriais e produtos relacionados (Politzer and Bon, 2006). As proteases são uma das principais enzimas com atuação industrial, junto com as amilases, lipases, celulases, xilanases e fitases (Mussatto et al., 2007).

Colagenases são proteases que fazem parte de um grupo de enzimas de importância vital, sobretudo, do ponto de vista fisiológico, atuando na manutenção e regeneração de órgãos e tecidos; além de sua extensa aplicabilidade biotecnológica. Enzimas que apresentam a propriedade colagenolítica também são capazes de hidrolisar as ligações peptídicas de vários tipos de colágeno. São de considerável importância na medicina humana e veterinária (Rao et al., 1998, Watanabe, 2004, Lima et al., 2015). Esta revisão objetivou realizar um levantamento quantitativo da produção cientifica relacionada a colagenase do pescado, bem como dissertar sobre as principais características bioquímicas e aplicações biotecnológicas dessas proteases, com ênfase em enzimas extraídas a partir de organismos aquáticos.

\section{Metodologia}

A presente revisão foi subdividida em dois momentos: 1. Levantamento da produção cientifica a partir do termo "collagenase fish" (colagenase de pescado), durante o período de 1963 a 2016, na base de dados Scopus (https://www.scopus.com/), identificando quais os principais anos, autores e tipos de documentos (incluem-se artigos, publicações em livros, capítulos ou outros, desde que correlacionadas com as palavras-chaves) quantificando o total de publicações; 2. Revisão bibliográfica sobre as principais características bioquímicas e aplicações biotecnológicas das colagenases, com ênfase nos segmentos alimentício, terapêutico e têxtil, a partir da base de dados Science Direct (http://www.sciencedirect.com/), PubMed (Http://www.ncbi.nlm.nih.gov/pubmed) e Google acadêmico (https://scholar.google.com.br/), sem restrição de período e tipo de documento.

\section{Evidências cientificas: Colagenase de pescado}

De acordo com a base de dados do Scopus, no período de 1963 até 2016, foram detectadas 274 citações ao termo "collagenase fish", referindo-se às colagenases de organismos aquáticos. De acordo com a figura 1 , o na ode 2012 foi o que apresentou o maior número de publicações, com 18 no total, representando $6,6 \%$ do total de publicações. Dentre os autores com maiores números de publicações, destaca-se Thomas $\mathrm{A}$. Braunbeck, com 2,19\% do total de publicações nesse mesmo período, como ilustrado na figura 2. No concernente aos principais meios de publicações, a revista Biochemistry, Genetics and Molecular Biology, com um total de 161 documentos, representando cerca de $58,8 \%$ do total de publicações, como ilustrado na figura 3 . Por fim, foi verificado que o entre os tipos de documentos, o principal meio de divulgação foi através de artigos, como ilustrado na figura 4, representando $90,9 \%$ do total de publicações acerca do tema. 


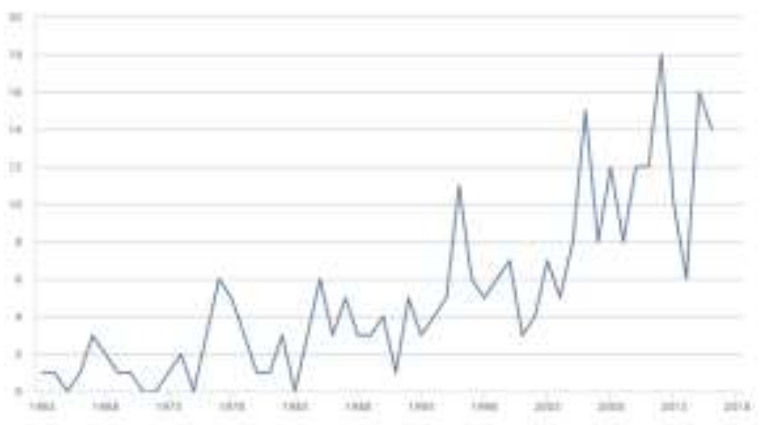

Figura 1. Produção cientifica relacionada ao termo "colagenase de pescado" no período de 1963 a 2016. Fonte: Scopus.

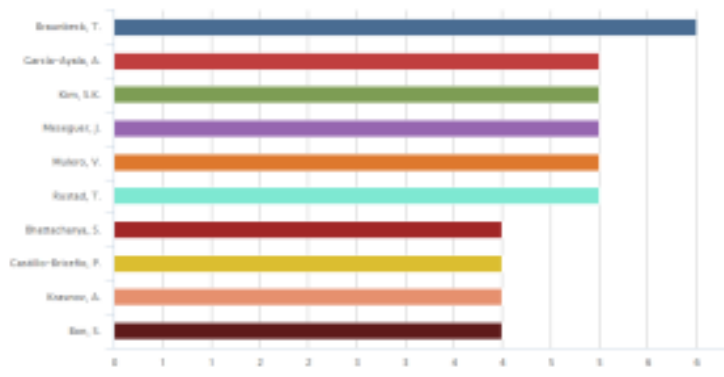

Figura 2. Produção cientifica por autor relacionado ao termo "colagenase de pescado" no período de 1963 a 2016. Fonte: Scopus.

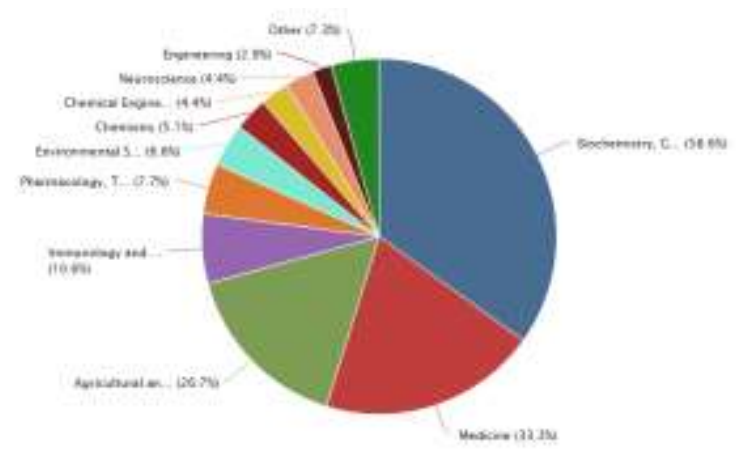

Figura 3. Produção cientifica por veículos de publicações relacionados ao termo "colagenase de pescado" no período de 1963 a 2016. Fonte: Scopus.

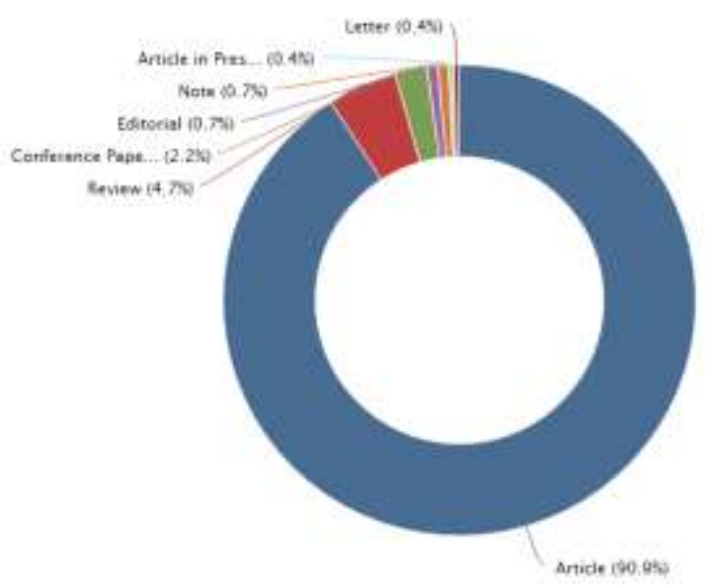

Figura 4. Principais tipos de divulgações relacionadas ao termo "colagenase de pescado" no período de 1963 a 2016. Fonte: Scopus.

\section{Características bioquímicas da colagenase}

Colagenases (EC 3.4.24.7) são um grupo de enzimas capazes de clivar o colágeno nativo ou desnaturado (Figura 5) em condições fisiológicas de $\mathrm{pH}$ e temperatura, in vivo e in vitro (Teruel and Simpson, 1995, Zimmer et al., 2009, Daboor et al., 2010), não apresentando atividade para qualquer outra proteína. Quando a molécula do colágeno é desnaturada, torna-se susceptível a hidrólise por outras proteases, como tripsina e quimotripsina, por exemplo, (Teruel and Simpson, 1995).

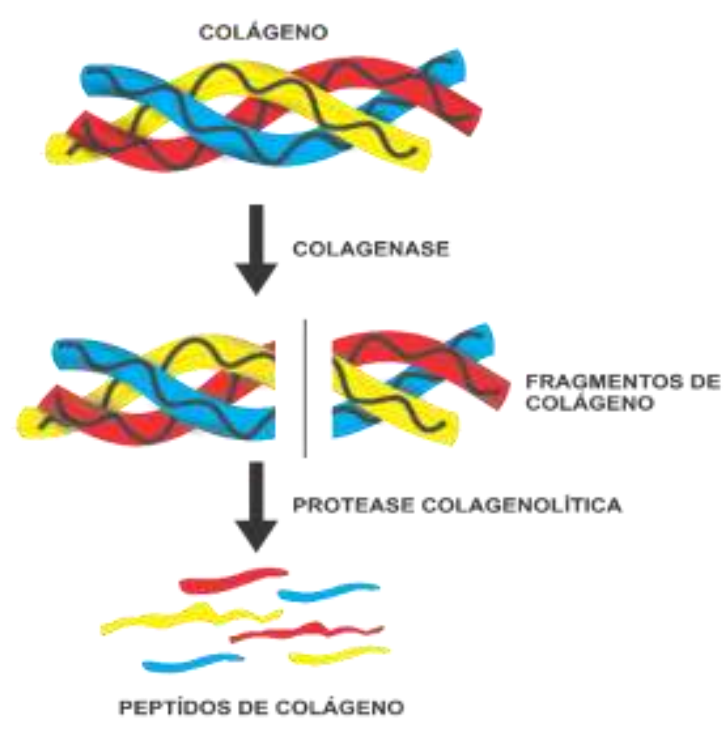

Figura 5. Clivagem do colágeno por protease colagenolítica.

A forma ativa da colagenase é obtida através da destruição de um inibidor ligado à enzima (endógeno) ou por clivagem de um zimogênio (Daboor et al., 2010). As metalocolagenases são secretadas como procolagenases (uma pró-enzima inativa), sendo necessário a atuação de ativadores, dentre os quais os mais utilizados para esta conversão são o 4-aminofenilmercúrico (Daboor et al., 2010), tripsina (Shinkai et al., 1977) e tiocianato de sódio (ou de potássio) (Daboor et al., 2012, Daboor et al., 2010).

Woessner (1977) observou que a tripsina reforçou a atividade da colagenase em quase $30 \%$. Por a tripsina ser considerada um ativador potente, supõe-se que a ativação do seu zimogênio (tripsinogênio) em sua forma ativa, indiretamente, possa ativar as colagenases presentes no meio. Ainda, foi proposto por Freitas-Júnior et al. (2012) um processo de aquecimento do extrato bruto enzimático (EBE) como forma de ativação do zimogênio e desnaturação de proteases que 
apresentem baixa resistência térmica, permanecendo, dessa forma, apenas as proteases resistentes a temperaturas elevadas por longos períodos de tempo. Dessa forma, teoriza-se que as proteases que apresentam a propriedade colagenolítica possam suportar o binômio tempo $\mathrm{x}$ temperatura por longo período.

A tabela 1 apresenta os parâmetros cinéticos e a sensibilidade a ativadores e inibidores de algumas colagenases de pescado. Em laboratório, as colagenases podem ser determinadas através de métodos colorimétricos, fluorescentes, radioativos e/ou viscosimétricos (Daboor et al., 2010). Os colorimétricos são os mais empregados, através do uso da ninhidrina (Daboor et al., 2010) e do azocoll em micro-organismos (Lima et al., 2015,

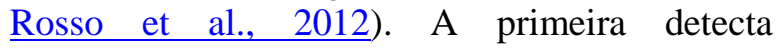
aminoácidos e peptídeos liberados pela degradação do colágeno (Park et al., 2002, Kim et al., 2002, Daboor et al., 2010), enquanto a segunda é um colágeno insolúvel que está ligado a um azocorante vermelho brilhante e tem sido utilizado na determinação da colagenolítica de proteases (Chavira et al., 1984, Lima et al., 2009, $\underline{\text { Rosso et }}$ al., 2012).

Tabela 1. Constante de Michaelis-Menten e sensibilidade a íons metálicos

\begin{tabular}{llccccl}
\hline Organismo & Nome cientifico & $\begin{array}{c}\mathrm{K}_{m} * \\
(\mathrm{Mm})\end{array}$ & $\begin{array}{c}V_{\text {máx }} * * \\
(\mathrm{U})\end{array}$ & Inibidores & Ativadores & Referência \\
\hline Micro-organismo & Bacillus licheniformis & 0.26 & 0.27 & $\mathrm{Fe}^{2+}, \mathrm{Mg}^{2+}, \mathrm{Mn}^{2+}$ & $\mathrm{Ca}^{2+}, \mathrm{Cu}^{2+}$ & $\underline{\text { Baehaki et al. (2012) }}$ \\
Pescado & Thunnus thymus & 3.82 & 851.5 & $\mathrm{Zn}^{2+}$ & $\mathrm{Li}^{+}, \mathrm{Na}^{+}, \mathrm{Ca}^{2+}$ & $\underline{\text { Byun et al. (2002) }}$ \\
Pescado & Novoden modestrus & $\mathrm{ND}$ & $\mathrm{ND}$ & $\mathrm{Hg}^{2+}, \mathrm{Zn}^{2+}, \mathrm{Ni}^{2+}$ & $\mathrm{Ba}^{2+}, \mathrm{Ca}^{2+}, \mathrm{K}^{+}$ & $\underline{\mathrm{Kim} \mathrm{et} \mathrm{al.} \mathrm{(2002)}^{2}}$ \\
Micro-organismo & Bacillus cereus & 1.31 & 12.54 & $\mathrm{Cu}^{2+}, \mathrm{Mn}^{2+}, \mathrm{Fe}^{2+}$ & $\mathrm{Ca}^{2+}, \mathrm{Zn}^{2+}, \mathrm{Mg}^{2+}$ & Liu et al. (2010) \\
Pescado & Scomber japonicus & 1.1 & 2.34 & $\mathrm{Hg}^{2+}, \mathrm{Zn}^{2+}$ & $\mathrm{Fe}^{2+}, \mathrm{Cd}^{2+}, \mathrm{Sn}^{2+}$ & $\underline{\text { Park et al. (2002) }}$ \\
\hline
\end{tabular}

* $\mathrm{K}_{m}=$ concentração de substrato na qual a reação acontece na metade da velocidade máxima;

*** $V_{\text {máx }}=$ velocidade máxima.

ND - não determinado.

Além dos inibidores metálicos, colagenases também são susceptíveis a inibidores naturais e sintéticos, tais como o $\beta$-mercaptoetanol, ácido etilenodiamino tetra-acético (EDTA) (Kristjánsson et al., 1995, Teruel and Simpson, 1995, Roy et al., 1996, Kim et al., 2002, Park et al., 2002, Daboor et al., 2010) e o inibidor de tripsina de soja (SBTI) (Daboor et al., 2012). Enzimas colagenolíticas podem ser extraídas a partir de uma variedade de técnicas (Figura 6) que utilizam diferentes sistemas de tampão (tris- $\mathrm{HCl}$, bicarbonato de sódio, cloreto de cálcio e cacodilato) (Daboor et al., 2012, Daboor et al., $\underline{2010})$.

O perfil eletroforético é muitas vezes empregado para estimar sua massa molar (Park et al., 2002, Kim et al., 2002, Wu et al., 2010a) que sofre variação em relação ao tipo de enzima e a sua origem (Byun et al., 2002, Park et al., 2002, Kim et al., 2002, Wu et al., 2010a, Daboor et al., 2012, Duarte et al., 2014).

\section{Fatores que afetam a atividade enzimática}

Proteases com propriedades colagenolíticas apresentam pequenas diferenças em suas características físico-químicas, quanto ao $\mathrm{pH}$ e a temperatura (Teruel and Simpson, 1995, Daboor et al., 2010). A tabela 2 ilustra as condições ótimas de $\mathrm{pH}$ e temperatura para colagenases do pescado em comparação com outras fontes (microorganismos e planta). Proteases colagenolíticas obtidas a partir de espécies de peixes e invertebrados exibem atividade ótima em $\mathrm{pH}$ fisiológico. Para a maioria das espécies aquáticas, esse $\mathrm{pH}$ situa-se numa faixa entre 6,0 e 8,0 (Park et al., 2002, Kim et al., 2002, Daboor et al., 2010). A estabilidade enzimática às variações de $\mathrm{pH}$ foi reportada para espécies aquáticas (peixes, crustáceos, esponjas), variando entre 5,0 a 10,0, a escala de recuperação de atividade (Kristjánsson et al., 1995, Teruel and Simpson, 1995, Lima et al., 2009, Mukherjee et al., 2009, Murado et al., 2009, Wu et al., 2010a, Hayet et al., 2011, Souchet and Laplante, 2011).

A temperatura é um fator limitador para proteases menos sensíveis e funciona como ativador de proteases que convertem procolagenases. Colagenases isoladas a partir de subprodutos de peixes marinhos têm evidenciado atividade em uma faixa de temperaturas, dependendo do tecido e das espécies das quais elas foram isoladas (Daboor et al., 2010), embora a faixa relatada até então esteja entre temperaturas $20{ }^{\circ} \mathrm{C}$ e $60{ }^{\circ} \mathrm{C}$ (Kim et al., 2002, Park et al., 2002, Daboor et al., 2012, Daboor et al., 2010, Wu et al., 2010a, Hayet et al., 2011). 


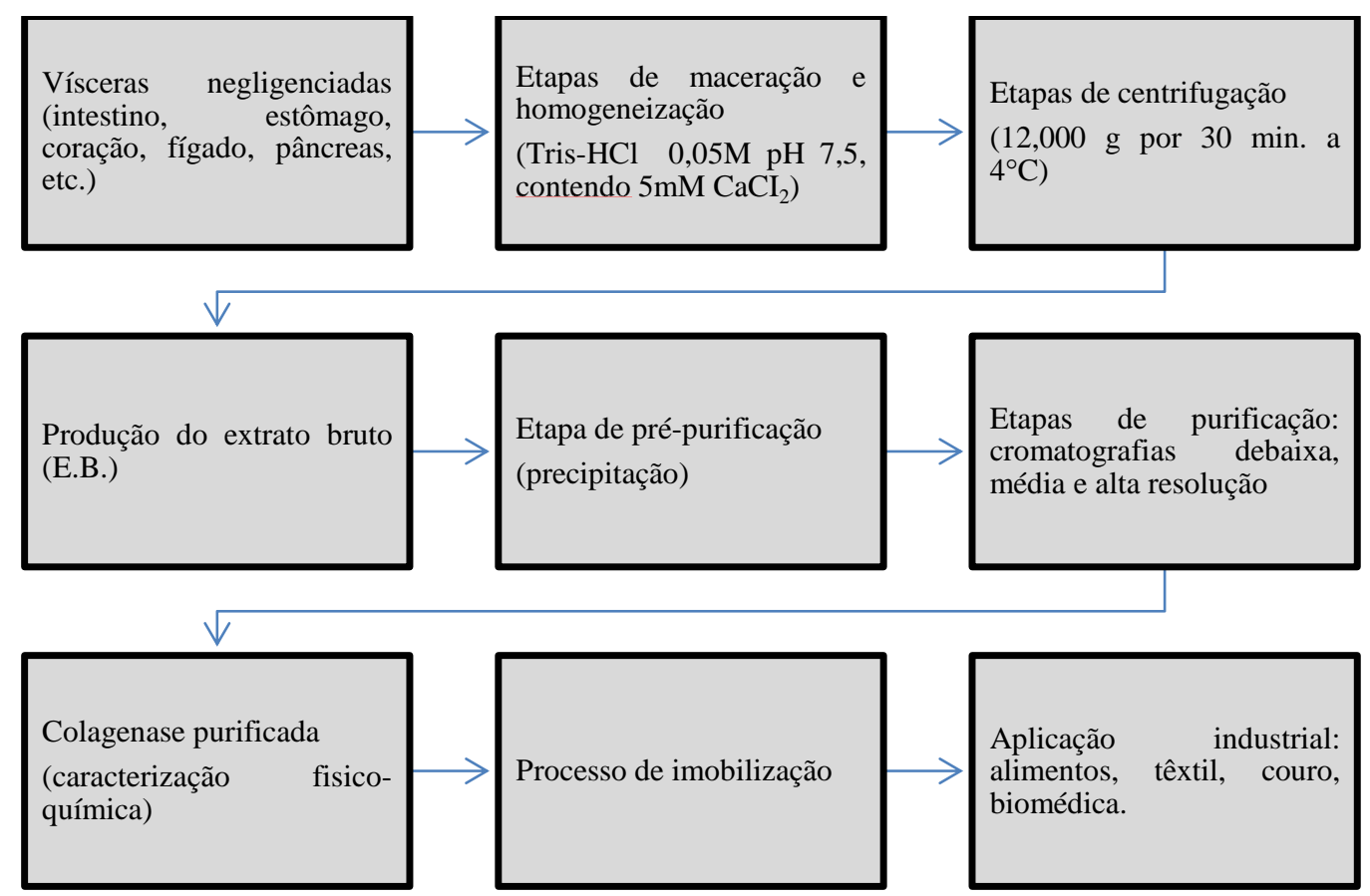

Figura 6: Etapas simplificadas para obtenção/extração de colagenase a partir de vísceras negligenciadas de peixes. No caso da indústria têxtil não se faz necessário que haja alto grau de purificação e imobilização, sendo dispensadas as demais etapas. A indústria farmacológica e de alimentos necessita de uma enzima com alto grau de pureza e que tenha passado por todos os testes antimicrobianos e antioxidantes, sendo necessárias todas as etapas (exceto a de imobilização, uma vez que é uma tecnologia altamente eficiente e desejada, porém não é indispensável).

Tabela 2. Fatores que afetam a atividade colagenolítica

\begin{tabular}{|c|c|c|c|c|}
\hline Organismo & Nome cientifico & $\mathrm{pH}$ & Temperatura & a Referência \\
\hline Peixe & Novoden modestrus & 8,0 & $55^{\circ} \mathrm{C}$ & Kim et al. (2002) \\
\hline Peixe & Pagrus major & 8,0 & $40^{\circ} \mathrm{C}$ & $\underline{\text { Wu et al. (2010a) }}$ \\
\hline Micro-organismo & Bacillus pumilus & 7,5 & $45^{\circ} \mathrm{C}$ & $\underline{\text { Wu et al. }(2010 \mathrm{~b})}$ \\
\hline Peixe & Sardinella aurita & 8,0 & $60^{\circ} \mathrm{C}$ & Hayet et al. (2011) \\
\hline Micro-organismo & Bacillus cereus & 8,0 & $40^{\circ} \mathrm{C}$ & $\underline{\text { Liu et al. (2010) }}$ \\
\hline Micro-organismo & Bacillus subtilis FS-2 & 9,0 & $50^{\circ} \mathrm{C}$ & Nagano and To (2000) \\
\hline Micro-organismo & Klebsiella pneumoniae & 6,0 & $37^{\circ} \mathrm{C}$ & $\underline{\text { Suphatharaprateep et al. (2011) }}$ \\
\hline Micro-organismo & Cândida albicans & 8,2 & $45^{\circ} \mathrm{C}$ & Lima et al. (2009) \\
\hline Micro-organismo & Bacillus licheniformis & 7,0 & $50^{\circ} \mathrm{C}$ & Baehaki et al. (2012) \\
\hline Planta & Ficus carica & 8,0 & $60^{\circ} \mathrm{C}$ & $\underline{\text { Raskovic et al. (2014) }}$ \\
\hline Micro-organismo & Bacillus stearothermophillus & 9,0 & $50^{\circ} \mathrm{C}$ & Lima et al. (2015) \\
\hline Esponja & Rhopaloeides odorabile & 5,0 & $30^{\circ} \mathrm{C}$ & Mukherjee et al. (2009) \\
\hline Peixe & Raja clavata & 6,0 & $40^{\circ} \mathrm{C}$ & Murado et al. (2009) \\
\hline Peixe & Gadus morhua & 8,0 & $50^{\circ} \mathrm{C}$ & Kristjánsson et al. (1995)e \\
\hline Caranguejo & Carcinus maenas & 7,0 & $30^{\circ} \mathrm{C}$ & $\underline{\text { Roy et al. (1996) }}$ \\
\hline Peixe & Pseudopleuronectes americanus & 7,5 & $40^{\circ} \mathrm{C}$ & Teruel and Simpson (1995) \\
\hline Peixe & Thunnus thymus & 7,5 & $55^{\circ} \mathrm{C}$ & Byun et al. (2002) \\
\hline Camarão & Macrobrachium rosenbergii & 7,0 & $60^{\circ} \mathrm{C}$ & $\underline{\text { Sriket et al. (2011) }}$ \\
\hline Peixe & Scomber japonicus & 7,5 & $55^{\circ} \mathrm{C}$ & $\underline{\text { Park et al. (2002) }}$ \\
\hline Camarão & Chionoecetes opilio & 8,0 & $40^{\circ} \mathrm{C}$ & $\underline{\text { Souchet and Laplante (2011) }}$ \\
\hline Mix de pescado & Mistura de resíduos (cabeça, barbatana, cauda etc.) & 7,5 & $35^{\circ} \mathrm{C}$ & Daboor et al. (2012) \\
\hline
\end{tabular}

\section{Fontes de colagenases}

A principal fonte de colagenase empregada na indústria é de origem microbiana a partir do Clostridium histolyticum (Teruel and Simpson,
1995, Kim et al., 2007, Duarte et al., 2014). Outras fontes microbianas já foram reportadas na literatura, como as obtidas a partir de $C$. albicans (Lima et al., 2009), Penicillium aurantiogriseum 
(Rosso et al., 2012, Lima et al., 2013), Bacillus pumilus (Wu et al., 2010b), B. cereus ((Liu et al., 2010) e B. licheniformis (Baehaki et al., 2012). A afinidade por vários sítios ao longo da cadeia de colágeno torna as colagenases de fontes microbianas uma das mais utilizadas. Apesar disso, a virulência de bactérias patogênicas produtoras deste tipo de enzima é uma grande desvantagem, à medida que suas aplicações são em locais de mobilização imunológica (Duarte et al., 2014).

Colagenases a partir de fontes vegetais também já foram citadas na literatura cientifica, tal como a descrita por Kim et al. (2007) e Raskovic et al. (2014). No entanto, espécies de peixes e invertebrados tem sido objeto de investigação por possuírem fontes vasta e disponíveis de colagenases e/ou de enzimas que apresentam a propriedade de clivar o colágeno nativo ou seus fragmentos, como as descritas por Teruel and Simpson (1995), Roy et al. (1996), Teruel (1997), Park et al. (2002), Kim et al. (2002), Wu et al. (2010a), Daboor et al. (2012).

Pelo reaproveitamento dos subprodutos do processamento da indústria do pescado, a colagenase tem sido investigada a nível de fígado, estômago, intestino, eventualmente parte anterior e posterior, dos cecos pilóricos, quando se fazem existentes, pâncreas ou do hepatopâncreas e da gordura mesentérica (Bertuzzi et al., 2009, Yoshinaka et al., 1976, Yoshinaka et al., 1977, Yoshinaka et al., 1978).

Yoshinaka et al. (1978) verificaram qual seria o local de produção de colagenase em diferentes órgãos que compõem o sistema digestivo (fígado, estômago, intestino delgado, mesentério) de diferentes espécies de peixes subtropicais, tais como nas espécies Sardinops melanosticta, Salmo gairdnerii, Cyprinus carpio, Parasilurus asotus, Anguilla japonica e Seriola quinquerdiota. Dentre as vísceras analisadas pelos autores, o estômago foi a que apresentou a menor atividade colagenolítica, quando comparado às demais vísceras, ou mesmo não demonstrou nenhuma atividade, dependendo da espécie. Uma das explicações para esse fato é a variabilidade morfológica do aparelho digestivo de peixes, diferindo das porções do intestino que são sempre ricas em colagenases. Soma-se a isso, o hábito alimentar, uma vez que espécies carnívoras tendem a necessidade de digerir o colágeno da alimentação (Seixas Filho, 2003).

\section{Classificação das colagenases}

Do ponto de vista fisiológico, as proteases colagenolíticas são agrupadas em dois grupos, com descritos na figura 7: (1) metalocolagenases, são as colagenases de vertebrados ou designadas de verdadeiras; e (2) serinocolagenases, às vezes chamadas de falsas colagenases, são as serinoproteases que exibem a característica de clivar a parte não helicoidal do colágeno.

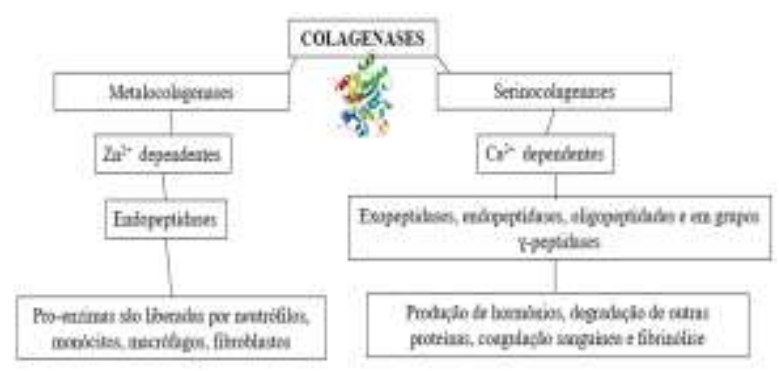

Figura 7: Classificação das colagenases.

\section{Metalocolagenases}

Metalocolagenases são endopeptidases da família das metaloproteinases (MMPs), que promovem a degradação da matriz extracelular, também conhecidas como matrixinas ou colagenases de vertebrados. Todos os membros dessa família são secretados como pró-enzimas ligadas à membrana (Cawston, 1996, Brinckerhoff and Matrisian, 2002, Greenlee et al., 2007, Murphy and Nagase, 2008, Tallant et al., 2010). Essas pró-enzimas são liberadas por neutrófilos, monócitos, macrófagos, fibroblastos e, além disso, também podem ser secretadas pelas células tumorais em resposta a uma variedade de estímulos, tais como processos cicatriciais e tumorais. Apresentam massas molares entre 30.000 e $150.000 \mathrm{Da}$. Como todos os membros das MMPs, são enzimas $\mathrm{Zn}^{2+}$ dependentes, sendo inibidas por qualquer quelante que se ligue a esse íon, tendo a necessidade do $\mathrm{Ca}^{2+}$ para manutenção da sua estabilidade (Jung and Winter, 1998, Daboor et al., 2012, Daboor et al., 2010). Apenas as MMP 1, 8, 13, 14 e 18 possuem a atividade de decompor os tipos de colágeno nativo de cadeia tripla I, II, III, VII e X (Daboor et al., 2010, Tallant et al., 2010, Araújo et al., 2011). São usualmente recuperadas de tecidos animais e de subprodutos do processamento do pescado, tais como ossos, barbatanas, peles e do hepatopâncreas de caranguejos marinhos (Bracho and Haard, 1995, Brinckerhoff and Matrisian, 2002, Daboor et al., 2010). 
Metaloproteinases podem ser divididas em: (I) colagenases verdadeiras, que clivam a tripla hélice do colágeno num único local ao longo das três cadeias, dando origem a produtos de 3/4 e 1/4 do comprimento da molécula original (MMP-1, 8 e 13); (II) gelatinases, que têm como alvo colágenos desnaturados e gelatinas (MMP-2 e 9); e (III) as estromelisinas, que têm uma ampla especificidade e podem degradar os proteoglicanos (MMP-3, 7 e 10), matrilisinas (MMP-7 e 26), MMPs tipo membrana (MMP-14, 15, 16, 17 e 24) e outras MMPs 3, que são classificadas pela especificidade ao substrato e, especialmente, de acordo com sua estrutura (Cawston, 1996, Greenlee et al., 2007, Murphy and Nagase, 2008, Tallant et al., 2010, Araújo et al., 2011). Enzimas dessa matriz participam de processos fisiológicos que envolvem reparação tecidual durante a implantação do blastocisto, ovulação, pós-parto e involução pós-lactação, a reabsorção óssea, cicatrização de feridas. Essa capacidade de processamento também é necessária durante a embriogênese e angiogênese (Tallant et al., 2010).

A participação das metaloproteinases em diversos eventos biológicos deve-se ao fato de que elas podem influenciar o comportamento celular através de algumas ações, como clivagem de proteínas que fazem a adesão célula-célula, liberação de moléculas bioativas na superfície celular ou por clivagem de moléculas presentes na superfície celular, as quais transmitem sinais no ambiente extracelular (Cawston, 1996, Araújo et al., 2011). Para que ocorra a catálise, são necessários substratos que incluem outras (pró)proteases, inibidores de protease, fatores de coagulação, peptídeos antimicrobianos, moléculas de adesão, fatores de crescimento, hormônios, citosinas, bem como os seus receptores e proteínas de ligação. Os substratos de metaloproteinases clivam o montante de resíduos hidrofóbicos, tipicamente leucina (Murphy and Nagase, 2008, Tallant et al., 2010). A desregulação das metaloproteinases pode dar origem à degradação e patologias decorrentes. Isto ocorre durante a inflamação, ulceração, artrite, periodontite, doença cardiovascular, fibrose e enfisema. Outras consequências da atividade metaloproteinase desregulamentada incluem processos associados ao câncer, como tumorigênese e neovascularização tumoral, invasão e metástase, assim como apoptose, ativação de defesa intestinal e patologias do sistema nervoso, tais como acidente vascular cerebral e doença de Alzheimer
(Murphy and Nagase, 2008, Tallant et al., 2010, Araújo et al., 2011).

\section{Serinocolagenases}

As serinocolagenases ou serinoproteases colagenolíticas, como todas as serinoproteases, contém um resíduo de serina no seu sítio catalítico. Estas enzimas são $\mathrm{Ca}^{2+}$ dependentes, apresentando massa molar variando entre 24.000 a $36.000 \mathrm{Da}$, estando muitas vezes associadas a órgãos digestivos, principalmente, intestinos e cecos (Yoshinaka et al., 1976, Yoshinaka et al., 1977, Yoshinaka et al., 1978, Kristjánsson et al., 1995, Kim et al., 2002, Park et al., 2002, Seixas Filho, 2003, Murado et al., 2009). Podem ser exopeptidases, endopeptidases, oligopeptidades e em grupos $\mathrm{\gamma}$-peptidases. São geralmente ativas em $\mathrm{pH}$ alcalino e neutro, com um $\mathrm{pH}$ ótimo entre 7 e 11 (Rao et al., 1998, Daboor et al., 2010).

Enzimas do grupo das serinocolagenases são capazes de clivar a tripla hélice do colágeno dos tipos I, II e III, e muitas vezes estão envolvidas com a produção de hormônios, degradação de outras proteínas, coagulação sanguínea e fibrinólise (Daboor et al., 2010). Serinoproteases colagenolíticas já foram isoladas a partir de vísceras digestivas, músculo e de resíduos gerais do processamento do pescado (aglomerado com cabeça, barbatanas, cauda, vísceras digestivas, reprodutivas), tais como os descritos por Turkiewicz et al. (1991) para Euphausia superba, Kristjánsson et al. (1995) para G. morhua, Teruel and Simpson (1995) para P. americanus, Roy et al. (1996) para C. maenas, Byun et al. (2002) para T. thymus, Kim et al. (2002) para o N. modestrus, Park et al. (2002) para S. japonicus, Mukherjee et al. (2009) para R. odorabile, Murado et al. (2009) para $R$. clavata, Wu et al. (2010a) para $P$. major, Hayet et al. (2011) para S. aurita, Souchet and Laplante (2011) para Chionoecetes opilio e Sriket et al. (2011) para Macrobrachium rosenbergii.

Vísceras digestivas de peixes são fontes tanto de serino quanto de metalocolagenases, todavia, a maioria dos estudos têm se concentrado em glândulas digestivas como fonte das colagenases portadoras do grupo serino. De tal modo, os resíduos de tecidos, além das glândulas digestivas, podem servir como um valioso e promissor manancial de moléculas biologicamente ativas para aplicações biotecnológicas (Daboor et al., $\underline{2010)}$. 


\section{Aplicações industriais da colagenase}

$\mathrm{Na}$ atualidade, vários processos biotecnológicos estão sendo utilizados para o desenvolvimento de soluções colagenolíticas que apresentem eficácia catalítica e baixo custo. $\mathrm{Na}$ indústria, colagenases são empregadas em diversas fases de produção e podem ser encontradas nos segmentos alimentício, terapêutico e têxtil. Watanabe (2004) classifica estas proteases em dois grupos/categorias distintas: (a) aquelas em que proteases colagenolíticas são utilizadas diretamente como em soluções tópicas a partir da adição direta em pomadas; e (b) aquelas em que são utilizados os produtos resultantes da sua reação, como os peptídeos bioativos gerados a partir da degradação de colágenos extraídos da pele de espécies de peixes, empregando-os na produção e aplicação de moléculas biologicamente ativas (Teruel, 1997).

\section{Aplicações na indústria de alimentos}

As colagenases são empregadas na indústria de alimentos nos segmentos de carne e pescado. Essas enzimas são adicionadas nas etapas de beneficiamento de produtos, tais como no preparo e amaciamento da carne, uma vez que o colágeno insolúvel contribui para a resistência da carne e dessa forma a enzima atua no processo de repartição do tecido conectivo aumentando a textura e a qualidade de carnes e derivados (Foegeding and Larick, 1986, Kim et al., 1993). No processamento do pescado, age na musculatura de peixes, auxiliando nas etapas de remoção da pele e extração do colágeno (Suphatharaprateep et al., 2011, Rochima et al., 2016). A patogenicidade de alguns tipos de bactérias e leveduras produtoras de colagenases é uma das preocupações da indústria alimentícia, uma vez que estirpes patogênicas podem contribuir para a virulência destes microorganismos e contaminar o alimento. Dessa forma, fontes alternativas para substituir as colagenases microbianas são altamente desejáveis pelo segmento alimentício. E uma das opções viáveis é a utilização de colagenases a partir de organismos aquáticos, como peixes, mariscos, camarões, esponjas, entre outros (Kim et al., 1993, Teruel, 1997).

\section{Aplicações na indústria biomédica e terapêutica}

A capacidade de decompor o colágeno sem afetar as propriedades de outras proteínas, tornou a colagenase uma ferramenta aplicável nas ciências médicas para limpar feridas necrosadas, escaras, cicatrizes pós-operatórias, em queimaduras, no tratamento de psoríase e pediculoses, em casos de implantes e perdas ósseas, em casos de lesões de mamilos de mulheres em aleitamento, no tratamento de cicatrizes hipertróficas, no tratamento de fibroma uterino, de isquemias do coração, de glaucoma, no reparo de cartilagem, de queloides, no de hérnia de disco intervertebral, no da celulite, no debridamento de úlceras de pessoas diabéticas e na regeneração dos tecidos da pele (Takahashi et al., 1999, Özcan et al., 2002, Watanabe, 2004, Arakawa et al., 2012, Alipour et al., 2016, Tallis et al., 2013, Brunengraber et al., 2014, Chandika et al., 2015).

Ainda, é empregada no tratamento das doenças de Peyronie, uma doença caracterizada pelo desenvolvimento de uma placa fibrótica ou de um nódulo que se instalam na túnica albugínea, estrutura que envolve os corpos cavernosos, comprometendo sua elasticidade e impedindo sua expansão (Levine, 2013, Langston and Carson, 2014); na doença de Dupuytren, uma contratura fixa da mão em flexão caracterizada pelo espessamento da fáscia palmar (tecido encontrado abaixo da pele da mão). Essa condição pode variar desde pequenos nódulos até faixas muito espessas, as quais podem tracionar dos dedos em direção à palma da mão (Martin-Ferrero et al., 2013, Peimer et al., 2013, Hentz, 2014, Meals and Hentz, 2014, Leclère et al., 2014), na osteoartrite (Adães et al., 2015), no tratamento de miomas uterinos (Jayes et al., 2016) e na remodelagem da matrix extracelular no câncer colorretal (Jucá et al., 2008).

Uma variedade de células produz colagenases, tais como neutrófilos, granulócitos, macrófagos, fibroblastos, queratinócitos. Dessa forma, a má regulação das proteases colagenolíticas é lesiva, principalmente quando já há algum agravo, uma vez que o colágeno é uma das principais proteínas da matriz. As células produtoras de colagenases desempenham um papel ativo nas diferentes fases de cicatrização de feridas. Os fibroblastos são atraídos para a ferida por fatores segregados a partir de macrófagos. Eles produzem colágeno novo para a reconstrução da matriz extracelular (Jung and Winter, 1998). As colagenases aumentam a proliferação, a angiogênese e a migração das células dérmicas, removendo os tecidos necróticos, mas não danificando o tecido saudável (Alipour et al., 2016). 
Existem inúmeras vantagens do uso de colagenases no tratamento de feridas: a) removem o tecido necrosado com maior eficiência por sua capacidade de hidrolisar vários tipos de colágeno; b) são indolores e não-hemorrágicos; c) podem ser usados por longos períodos e também em associação com outros medicamentos; d) atraem macrófagos e fibroblastos para o local da ferida; e) aumentam a formação de tecidos de granulação e estimulam o próprio organismo a promover a cicatrização (Jung and Winter, 1998).

Na medicina veterinária, já foram empregadas para quebrar a rede fibromuscular para isolamento de células espermatogênicas do tecido testicular em equinos (Jung and Yoon, 2016), em casos de tendinite equina (Wallis et al., 2010, Oloumi et al., 2011) e no isolamento de células-tronco mesenquimais em caninos (Lee et al., 2013).

\section{Aplicações na indústria têxtil e do couro}

Proteases com propriedades colagenolíticas têm sido aplicadas no tratamento de couros e tingimento de tecidos, por funcionar como um biocatalisador não tóxico, melhorando as características de tingimento, além de se tornar um processo ecologicamente viável (eco-benigno) por reduzir a carga poluidora no processo póstingimento. Materiais não colagenosos indesejados são removidos durante o processamento de couro. O processamento do couro envolve várias operações unitárias de précurtimento, curtimento, pós-bronzeamento e acabamento. Couros tratados com colagenase resultam em tingimento uniforme, brilhante e com solidez comparável aos tratamentos não enzimáticos (Kanth et al., 2008).

\section{Considerações finais}

Colagenases são enzimas de valor precioso para a indústria. Seu emprego nos diversos segmentos - alimentício, têxtil, terapêutico, por exemplo -, está cada vez mais abrangente. Além das qualidades bioquímicas das colagenases de organismos aquáticos, a redução da carga de poluentes é uma das vantagens de se fazer uso de enzimas dessa fonte, visando tornar os processos produtivos ecologicamente sustentável e viável (eco-benigno). Assim, fontes alternativas de colagenases são ao mercado um produto novo, de qualidade considerável e com perspectiva de redução da carga poluidora, agregando valor ao produto da pesca e impulsionando a economia.

\section{Referências Bibliográficas}

Adães, S., Ferreira-Gomes, J., Mendonca, M., Almeida, L., Castro-Lopes, J. M. \& Neto, F. L. 2015. Injury of primary afferent neurons may contribute to osteoarthritis induced pain: an experimental study using the collagenase model in rats. Osteoarthritis and Cartilage, 23, 914-924.

Alipour, H., Raz, A., Zakeri, S. \& Djadid, N. D. 2016. Therapeutic applications of collagenase (metalloproteases): A review. Asian Pacific Journal of Tropical Biomedicine, 6, 975-981.

Arakawa, H., Uehara, J., Hara, E. S., Sonoyama, W., Kimura, A., Kanyama, M., Matsuka, Y. \& Kuboki, T. 2012. Matrix metalloproteinase- 8 is the major potential collagenase in active periimplantitis. Journal of Prosthodontic Research, 56, 249-255.

Araújo, R. V. S., Silva, F. O., Melo-Júnior, M. R. \& Porto, A. L. F. 2011. Metaloproteinases: aspectos fisiopatológicos sistêmicos e sua importância na cicatrização. Revista de Ciências Médicas e Biológicas, 10, 82-88.

Baehaki, A., Suhartono, M. T., Syah, D., Sitanggang, A. B., Setyahadi, S. \& Meinhardt, F. 2012. Purification and characterization of collagenase from Bacillus licheniformis F11. 4. African Journal of Microbiology Research, 6, 2373-2379.

Bertuzzi, F., Cainarca, S., Marzorati, S., Bachi, A., Antonioli, B., Nano, R., Verzaro, R. \& Ricordi, C. 2009. Collagenase isoforms for pancreas digestion. Cell Transplantation, 18, 203-206.

Bracho, G. E. \& Haard, N. F. 1995. Identification of two matrix metalloproteinases in the skeletal muscle of Pacific rockfish (Sebastes sp.). Journal of Food Biochemistry, 19, 299-319.

Brinckerhoff, C. E. \& Matrisian, L. M. 2002. Matrix metalloproteinases: a tail of a frog that became a prince. Nature Reviews Molecular Cell Biology, 3, 207-214.

Brunengraber, L. N., Jayes, F. L. \& Leppert, P. C. 2014. Injectable Clostridium histolyticum collagenase as a potential treatment for uterine fibroids. Reproductive Sciences, 21, 14521459 .

Bussiness Standard. 2016. Global enzymes market to reach $\$ 7.2$ billion in 2020: Report. Disponível em: <http://www.businessstandard.com/content/b2b-chemicals/global- 
enzymes-market-to-reach-7-2-billion-in-2020report-116062301172_1.html>. Acesso em: 11 de dez. 2016.

Byun, H. G., Park, P. J., Sung, N. I. \& Kim, S. K. 2002. Purification and characterization of a serine proteinase from the tuna pyloric caeca. Journal of Food Biochemistry, 26, 479-494.

Cawston, T. E. 1996. Metalloproteinase inhibitors and the prevention of connective tissue breakdown. Pharmacology \& Therapeutics, 70, 163-182.

Chandika, P., Ko, S.-C., Oh, G.-W., Heo, S.-Y., Nguyen, V.-T., Jeon, Y.-J., Lee, B., Jang, C. H., Kim, G. \& Park, W. S. 2015. Fish collagen/alginate/chitooligosaccharides integrated scaffold for skin tissue regeneration application. International journal of biological macromolecules, 81, 504-513.

Chavira, R., Burnett, T. J. \& Hageman, J. H. 1984. Assaying proteinases with azocoll. Analytical Biochemistry, 136, 446-450.

Daboor, S. M., Budge, S. M., Ghaly, A. E., Brooks, M. S. \& Dave, D. 2012. Isolation and activation of collagenase from fish processing waste. Advances in Bioscience and Biotechnology, 3, 191-209.

Daboor, S. M., Budge, S. M., Ghaly, A. E., Brooks, S. L. \& Dave, D. 2010. Extraction and purification of collagenase enzymes: a critical review. American Journal of Biochemistry and Biotechnology, 6, 239-263.

Duarte, K. F., Junqueira, O. M., Faria Domingues, C. H., Silva Filardi, R., Borges, L. L. \& Praes, M. F. F. M. 2014. Digestible valine requirements for broilers from 22 and 42 days old. Acta Scientiarum. Animal Sciences, 36, 151-156.

Foegeding, E. A. \& Larick, D. K. 1986. Tenderization of beef with bacterial collagenase. Meat Science, 18, 201-214.

Freitas-Júnior, A. C. V., Costa, H. M. S., Icimoto, M. Y., Hirata, I. Y., Marcondes, M., Carvalho, L. B., Oliveira, V. \& Bezerra, R. S. 2012. Giant Amazonian fish pirarucu (Arapaima gigas): its viscera as a source of thermostable trypsin. Food Chemistry, 133, 1596-1602.

Greenlee, K. J., Werb, Z. \& Kheradmand, F. 2007. Matrix metalloproteinases in lung: multiple, multifarious, and multifaceted. Physiological Reviews, 87, 69-98.
Hayet, B. K., Rym, N., Ali, B., Sofiane, G. \& Moncef, N. 2011. Low molecular weight serine protease from the viscera of sardinelle (Sardinella aurita) with collagenolytic activity: Purification and characterisation. Food Chemistry, 124, 788-794.

Hentz, V. R. 2014. Collagenase injections for treatment of Dupuytren disease. Hand Clinics, 30, 25-32.

Jayes, F. L., Liu, B., Moutos, F. T., Kuchibhatla, M., Guilak, F. \& Leppert, P. C. 2016. Loss of stiffness in collagen-rich uterine fibroids after digestion with purified collagenase Clostridium histolyticum. American Journal of Obstetrics and Gynecology, 215, 596.e1596.e8.

Jucá, M., Nunes, B. L. B. B. P., Menezes, H. L., Gomes, E. G. A. \& Matos, D. 2008. Metaloproteinases 1 e 7 e câncer colorretal. Revista Brasileira de Coloproctologia, 28, 353-362.

Jung, H. \& Yoon, M. 2016. Isolation of germ cells from testes of stallions using collagenase and trypsin-ethylenediaminetetraacetic acid. Journal of Equine Veterinary Science, 43, 8287.

Jung, W. \& Winter, H. 1998. Considerations for the use of Clostridial collagenase in clinical practice. Clinical Drug Investigation, 15, 245252.

Kanth, S. V., Venba, R., Madhan, B., Chandrababu, N. K. \& Sadulla, S. 2008. Studies on the influence of bacterial collagenase in leather dyeing. Dyes and Pigments, 76, 338-347.

Kim, M., Hamilton, S. E., Guddat, L. W. \& Overall, C. M. 2007. Plant collagenase: Unique collagenolytic activity of cysteine proteases from ginger. Biochimica et Biophysica Acta (BBA)-General Subjects, 1770, 1627-1635.

Kim, S.-K., Byun, H.-G., Choi, K.-D., Roh, H.-S., Lee, W.-H. \& Lee, E.-H. 1993. Removal of skin from filefish using enzymes. Korean Journal of Fisheries and Aquatic Sciences, 26, 159-172.

Kim, S.-K., Park, P.-J., Kim, J.-B. \& Shahidi, F. 2002. Purification and characterization of a collagenolytic protease from the filefish, Novoden modestrus. Journal of Biochemistry and Molecular Biology, 35, 165-171. 
Kristjánsson, M. M., Gudmundsdóttir, S., Fox, J. W. \& Bjarnason, J. B. 1995. Characterization of a collagenolytic serine proteinase from the Atlantic cod (Gadus morhua). Comparative Biochemistry and Physiology Part B: Biochemistry and Molecular Biology, 110, 707-717.

Langston, J. P. \& Carson, C. C. 2014. Peyronie's disease: review and recent advances. Maturitas, 78, 341-343.

Leclère, F. M., Mathys, L. \& Vögelin, E. 2014. Traitement de la maladie de Dupuytren par collagénase injectable, évaluation de l'échographie assistée. Chirurgie de la Main, 33, 196-203.

Lee, K. S., Nah, J. J., Lee, B. C., Lee, H. T., Lee, H. S., So, B. J. \& Cha, S. H. 2013. Maintenance and characterization of multipotent mesenchymal stem cells isolated from canine umbilical cord matrix by collagenase digestion. Research in Veterinary Science, 94, 144-151.

Levine, L. A. 2013. Peyronie's disease: A contemporary review of non-surgical treatment. Arab Journal of Urology, 11, 278283.

Lima, C. A., Campos, J. F., Lima Filho, J. L., Converti, A., da Cunha, M. G. C. \& Porto, A. L. F. 2015. Antimicrobial and radical scavenging properties of bovine collagen hydrolysates produced by Penicillium aurantiogriseum URM 4622 collagenase. Journal of Food Science and Technology, 52, 4459-4466.

Lima, C. A., Freitas Júnior, A. C. V., Lima Filho, J. L., Converti, A., Marques, D. A. V., Carneiro-da-Cunha, M. G. \& Porto, A. L. F. 2013. Two-phase partitioning and partial characterization of a collagenase from Penicillium aurantiogriseum URM4622: Application to collagen hydrolysis. Biochemical Engineering Journal, 75, 64-71.

Lima, C. A., Rodrigues, P. M. B., Porto, T. S., Viana, D. A., Lima Filho, J. L., Porto, A. L. F. \& Cunha, M. G. C. 2009. Production of a collagenase from Candida albicans URM3622. Biochemical Engineering Journal, 43, 315320.

Liu, L., Ma, M., Cai, Z., Yang, X. \& Wang, W. 2010. Purification and properties of a collagenolytic protease produced by Bacillus cereus MBL13 strain. Food Technology \& Biotechnology, 48.

Martin-Ferrero, M. A., Simón-Pérez, C., Rodríguez-Mateos, J. I., García-Medrano, B., Hernández-Ramajo, R. \& Brotat-García, M. 2013. Treatment of Dupuytren's disease using collagenase from Clostridium histolyticum. Revista Española de Cirugía Ortopédica y Traumatología, 57, 398-402.

Meals, R. A. \& Hentz, V. R. 2014. Technical tips for collagenase injection treatment for Dupuytren contracture. The Journal of Hand Surgery, 39, 1195-1200.

Mukherjee, J., Webster, N. \& Llewellyn, L. E. 2009. Purification and characterization of a collagenolytic enzyme from a pathogen of the Great Barrier Reef sponge, Rhopaloeides odorabile. PLoS One, 4, e7177.

Murado, M. A., González, M. d. P. \& Vázquez, J. A. 2009. Recovery of proteolytic and collagenolytic activities from viscera byproducts of rayfish (Raja clavata). Marine Drugs, 7, 803-815.

Murphy, G. \& Nagase, H. 2008. Progress in matrix metalloproteinase research. Molecular Aspects of Medicine, 29, 290-308.

Mussatto, S. I., Fernandes, M. \& Milagres, A. M. F. 2007. Enzimas: Poderosa ferramenta na indústria. Ciência Hoje, 242, 27-33.

Nagano, H. \& To, K. A. 2000. Purification of collagenase and specificity of its related enzyme from Bacillus subtilis FS-2. Bioscience, Biotechnology, and Biochemistry, 64, 181-183.

Oloumi, M. M., Vosough, D., Derakhshanfar, A. \& Nematollahi, M. H. 2011. The healing potential of Plantago lanceolata ointment on collagenase-induced tendinitis in burros (Equus asinus). Journal of Equine Veterinary Science, 31, 470-474.

Özcan, C., Ergün, O., Çelik, A., Çördük, N. \& Özok, G. 2002. Enzymatic debridement of burn wound with collagenase in children with partial-thickness burns. Burns, 28, 791-794.

Park, P.-J., Lee, S.-H., Byun, H.-G., Kim, S.-H. \& Kim, S.-K. 2002. Purification and characterization of a collagenase from the mackerel, Scomber japonicus. Journal of Biochemistry and Molecular Biology, 35, 576582. 
Peimer, C. A., Skodny, P. \& Mackowiak, J. I. 2013. Collagenase clostridium histolyticum for Dupuytren contracture: patterns of use and effectiveness in clinical practice. The Journal of Hand Surgery, 38, 2370-2376.

Politzer, K. \& Bon, E. P. S. 2006. Enzimas industriais e especiais. Ciência.

Rao, M. B., Tanksale, A. M., Ghatge, M. S. \& Deshpande, V. V. 1998. Molecular and biotechnological aspects of microbial proteases. Microbiology and Molecular Biology Reviews, 62, 597-635.

Raskovic, B., Bozovic, O., Prodanovic, R., Niketic, V. \& Polovic, N. 2014. Identification, purification and characterization of a novel collagenolytic serine protease from fig (Ficus carica var. Brown Turkey) latex. Journal of Bioscience and Bioengineering, 118, 622-627.

Rochima, E., Sekar, N., Buwono, I. D., Afrianto, E. \& Pratama, R. I. 2016. Isolation and characterization of collagenase from Bacillus subtilis (Ehrenberg, 1835); ATCC 6633 for degrading fish skin collagen waste from Cirata Reservoir, Indonesia. Aquatic Procedia, 7, 7684.

Rosso, B. U., Albuquerque, L. C., Porto, T. S., Oliveira, N. C., Pessoa, A., Converti, A., Graças, M. C.-d.-C. \& Porto, A. L. F. 2012. Partitioning and extraction of collagenase from Penicillium aurantiogriseum in poly (ethylene glycol)/phosphate aqueous two-phase system. Fluid Phase Equilibria, 335, $20-25$.

Roy, P., Colas, B. \& Durand, P. 1996. Purification, kinetical and molecular characterizations of a serine collagenolytic protease from greenshore crab (Carcinus maenas) digestive gland. Comparative Biochemistry and Physiology Part B: Biochemistry and Molecular Biology, 115, 8795.

Seixas Filho, J. T. 2003. Revisão sobre as enzimas digestivas em peixes teleósteos e seus métodos de determinação. Augustus, 8, 30-45.

Shinkai, H., Kawamoto, T., Hori, H. \& Nagal, Y. 1977. A complex of collagenase with low molecular weight inhibitors in the culture medium of embryonic chick skin explants. The Journal of Biochemistry, 81, 261-263.

Souchet, N. \& Laplante, S. 2011. Recovery and characterization of a serine collagenolytic extract from snow crab (Chionoecetes opilio) by-products. Applied Biochemistry and Biotechnology, 163, 765-779.

Sriket, C., Benjakul, S., Visessanguan, W. \& Kishimura, H. 2011. Collagenolytic serine protease in fresh water prawn (Macrobrachiumrosenbergii): Characteristics and its impact on muscle during iced storage. Food Chemistry, 124, 29-35.

Suphatharaprateep, W., Cheirsilp, B. \& Jongjareonrak, A. 2011. Production and properties of two collagenases from bacteria and their application for collagen extraction. New Biotechnology, 28, 649-655.

Takahashi, S., Geenen, D., Nieves, E. \& Iwazumi, T. 1999. Collagenase degrades collagen in vivo in the ischemic heart. Biochimica et Biophysica Acta (BBA)-General Subjects, 1428, 251-259.

Tallant, C., Marrero, A. \& Gomis-Rüth, F. X. 2010. Matrix metalloproteinases: fold and function of their catalytic domains. Biochimica et Biophysica Acta (BBA)-Molecular Cell Research, 1803, 20-28.

Tallis, A., Motley, T. A., Wunderlich, R. P., Dickerson, J. E., Waycaster, C. \& Slade, H. B. 2013. Clinical and economic assessment of diabetic foot ulcer debridement with collagenase: results of a randomized controlled study. Clinical Therapeutics, 35, 1805-1820.

Teruel, S. R. L. \& Simpson, B. K. 1995. Characterization of the collagenolytic enzyme fraction from winter flounder (Pseudopleuronectes americanus). Comparative Biochemistry and Physiology Part B: Biochemistry and Molecular Biology, 112, 131-136.

Teruel, S. R. L. T. 1997. Purification and cbaractefuzation of collagenases from the skeletal muscle of winter flounder (Pseudopleuronectes americanus). Department of Food Science and Agricultural Chemistry Macdonald Campus. McGill University Montreal, Quebec, Montreal.

Turkiewicz, M., Galas, E. \& Kalinowska, H. 1991. Collagenolytic serine proteinase from Euphausia superba Dana (Antarctic krill). Comparative Biochemistry and Physiology Part B: Comparative Biochemistry, 99, 359371.

Wallis, T. W., Baxter, G. M., Werpy, N. M., Mason, G. L., Frisbie, D. D. \& Jarloev, N. 2010. Acellular urinary bladder matrix in a 
collagenase model of superficial digital flexor tendonitis in horses. Journal of Equine Veterinary Science, 30, 365-370.

Watanabe, K. 2004. Collagenolytic proteases from bacteria. Applied Microbiology and Biotechnology, 63, 520-526.

Woessner, J. F. 1977. A latent form of collagenase in the involuting rat uterus and its activation by a serine proteinase. Biochemical Journal, 161, 535-542.

Wu, G.-P., Chen, S.-H., Liu, G.-M., Yoshida, A., Zhang, L.-J., Su, W.-J. \& Cao, M.-J. 2010a. Purification and characterization of a collagenolytic serine proteinase from the skeletal muscle of red sea bream (Pagrus major). Comparative Biochemistry and Physiology Part B: Biochemistry and Molecular Biology, 155, 281-287.

Wu, Q., Li, C., Li, C., Chen, H. \& Shuliang, L. 2010b. Purification and characterization of a novel collagenase from Bacillus pumilus ColJ. Applied Biochemistry and Biotechnology, 160, 129-139.

Yoshinaka, R., Sato, M. \& Ikeda, S. 1976. Studies on collagenase in fish, 2: Some properties of a collagenase from the pyloric caeca of Seriola

Zimmer, K. R., Borré, G. L., Trentin, D. S., Júnior, C. W., Frasson, A. P., Graeff, A. A., Gomes, P. \& Macedo, A. J. 2009. Enzimas microbianas de uso terapêutico e diagnóstico clínico. Revista Liberato, 10, 123-137. quinqueradiata. Bulletin of the Japanese Society of Scientific Fisheries, 42, 42, 455463.

Yoshinaka, R., Sato, M. \& Ikeda, S. 1977. Studies on collagenase in fish. III. Purification and properties of a collagenase from the pyloric caeca of yellow-tail. Bulletin of the Japanese Society for the Science of Fisheries, 43, 11951201.

Yoshinaka, R., Sato, M. \& Ikeda, S. 1978. Distribution of collagenase in the digestive organs of some teleosts. Bulletin of the Japanese Society for the Science of Fisheries, 44, 263-267.

\section{Article History:}

Received 19 December 2016

Accepted 18 January 2016

Available on line 14 February 2017

License information: This is an open-access article distributed under the terms of the Creative Commons Attribution License 4.0, which permits unrestricted use, distribution, and reproduction in any medium, provided the original work is properly cited. 